\title{
GREENHOUSE WEEDS IN THE BOTANICAL GARDEN OF PAS IN WARSAW-POWSIN
}

\author{
HALINA GALERA, HALinA RATYŃSKA \\ Botanical Garden - Center for Biological Diversity, Conservation of the Polish Academy of Sciences \\ Prawdziwka 2, 02-973 Warszawa
}

(Received: November 19, 1998, Accepted: June 20, 1999)

\begin{abstract}
Systematic studies carried out in the greenhouses of the Botanical Garden - Center for Biological Diversity Conservation of the Polish Academy of Sciences in Warsaw-Powsin revealed that the flora of weeds ras very rich there. A total of 243 taxa of vascular plants and 17 species of bryophytes were recorded. With regard to the division of the flora of vascular plants according to species origin and degree of naturalization, it was found that native plants ( 89 species), greenhouse-escapes ( 87 species) and permanently established aliens (48 species) co-dominated there. In addition, 10 species of garden-escapes, i.e. plants cultivated in the immediate neighbourhood of greenhouses, were recorded indoors. Noteworthy were two species that had been brought accidentally to the glasshouses together with plant material - this group of plants is least numerous but quite frequent in the study area. Observations made in the vicinity of Powsin greenhouses showed that relationships between the studied flora and the plants growing outside were unilateral. In Powsin no proved example of expansion of greenhouse plants to other parts of the Garden has been recorded. By contrast, influx of diaspores to the study area is observed frequently - the flora of greenhouse weeds is dominated by plants found also in the immediate neighbourhood ( 147 species). The most important factors affecting the composition of the weed flora of glasshouses include the flora of the surrounding areas and the mode of greenhouse management (e.g. the range of species cultivated there). The diversity of the flora is not related to greenhouse size.
\end{abstract}

KEY WORDS: synanthropic flora, weeds, floristic analysis, Botanical Garden of the Polish Academy of Sciences.

\section{INTRODUCTION}

Greenhouses are usually associated with horticulture and exhibition of exotic plants. Thus, they are centres of introduction of alien species (originating from warmer climatic zones) and potentially may become sources of spontaneous dispersion of some taxa. Because of this, they are an extremely interesting, although unappreciated, subject of research. Hereto only one paper on vascular plants growing as weeds in productive greenhouses has been published in Poland (Wiśniewski, Asprou 1974). Short notes were made in monographs on the flora of the Botanical Garden in Moscow (Kozhevnikov 1935) and on the flora of the city of Kraków (TrzcińskaTacik 1971 and 1979). These were, however, incidental data, not based on systematic observations. Similarly, there are no comparative data on the greenhouse flora of bryophytes. The reports published to date have been concerned exclusively with alien species (Podpera 1949a,b; Pilous 1956; Menzel 1984; Rusińska, Ratyńska, Galera 1996). A more complete study of bryophytes of the Wrocław Botanical Garden (Berdowski 1988) did not include the area inside greenhouses. The same applies to publications by Filipiak (1996), who did not take into account the area of tunnel houses in the Botanical Garden in Łódź.

This paper concerns the area of exhibition greenhouses and of the greenhouses used for plant propagation. The purpose of this study was to identify and analyse the spontaneous flora accompanying the plants cultivated in the greenhouses of the Botanical Garden - Centre for Biological Diversity Conservation of the Polish Academy of Sciences in Powsin near Warsaw. Vascular plants and bryophytes were studied, including both the weeds introduced accidentally to greenhouses and exotic plants that escaped from cultivation.

\section{STUDY AREA AND OBJECT OF INVESTIGATION}

The study area is a complex of glasshouses (composed of a corridor and twelve separate rooms called chambers), covering an area of $2382 \mathrm{~m}^{2}$, and a small one-chamber glasshouse of $92 \mathrm{~m}^{2}$ located at the back of the Gardens (Fig. 1). Plants are cultivated in pots, in patches (i.e. in the ground) at the floor level and in so-called windowsills, i.e. metal trays located about $0.7 \mathrm{~m}$ above the ground. Some epiphytes are exposed on branches covered with peat moss. There is also a small water body. In the corridor, potting compost is stored. The modernized chambers 5 and 6 are distinguished by the lack of windowsills, and by the presence of supports for epiphytes.

Apart from the permanent exhibitions of exotic plants, the greenhouses are also the place where plants for outdoor collections are propagated and some sensitive plant specimens are kept during the winter season. 


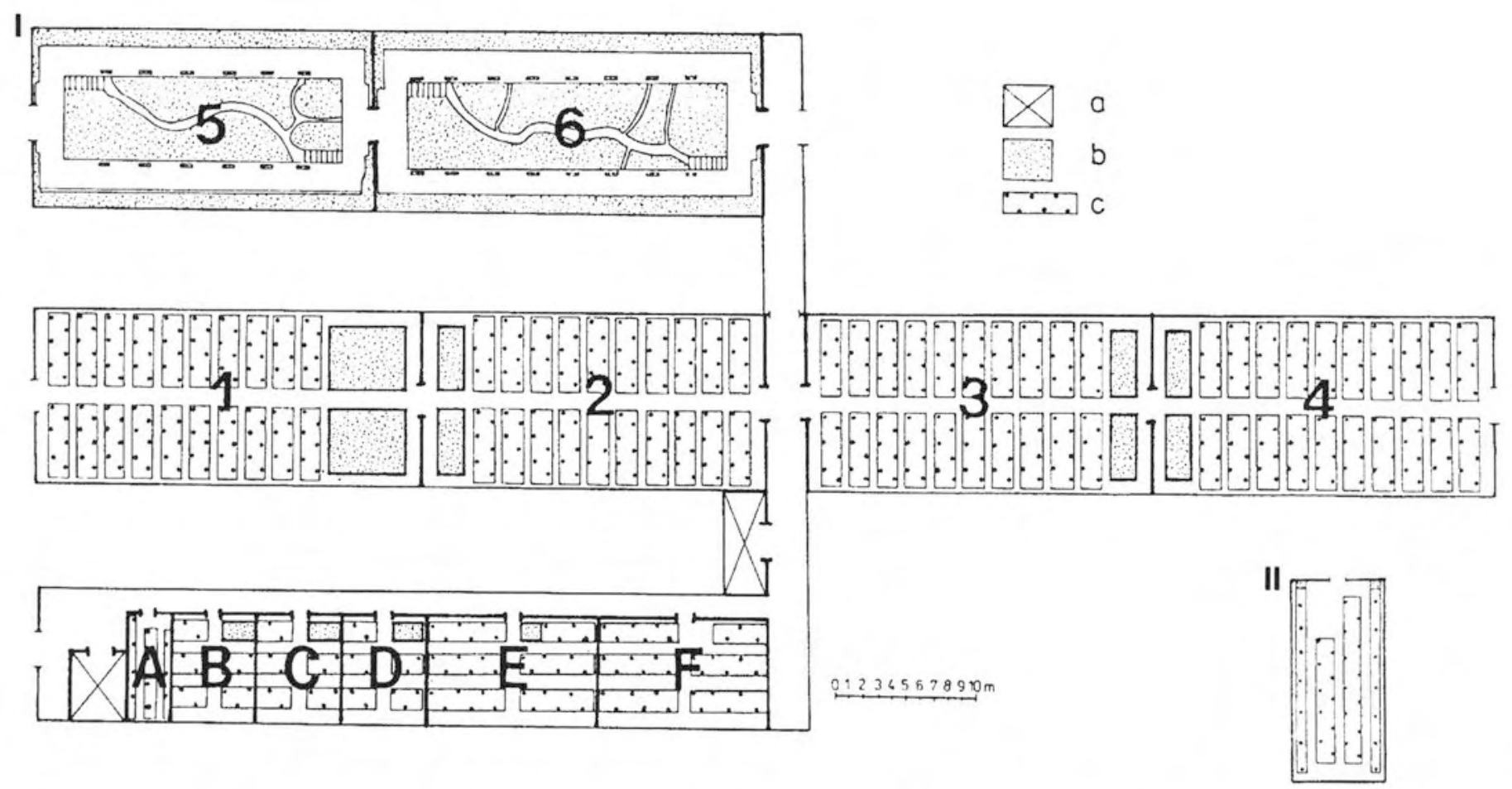

Fig. 1. Greenhouses in Botanical Garden in Powsin. I - complex of glasshouses, II - one-chamber glasshouse; a - store, b-patches in the ground, $\mathrm{c}-$ windowsills.

Greénhouses are a peculiar study area because of the specific climatic and soil conditions, and because of the pursued agronomic practices. Air temperature and water content are much higher than outside, while exposure to sunlight is much lower. The soil comes from various sources and is highly diverse in terms of mechanical and chemical composition. The substrate for plants is a mixture of compost (produced in the Garden), and sand, bark or peat (purchased from the outside). In the course of substrate preparation, the components are usually not exposed to thermic or chemical sterilization. Most important of the practices pursued in the greenhouses include systematic weeding, application of liquid fertilizers and spraying with pesticides. In a relatively small area, about 1700 taxa have been collected, with thousands of specimens, which results in increased competition between them. Characteristic are also marked differences in conditions between the chambers (Table 1).

TABLE 1. Greenhouses characteristic.

\begin{tabular}{l|c|c|c|l}
\hline Chambers & Year of building & Area $\left[\mathrm{m}^{2}\right]$ & Min. temp. $\left[{ }^{\circ} \mathrm{C}\right]$ & Most important permanent collections \\
\hline 1 & 1980 & 324.0 & $20-22$ & tropical ferns, Ficus \\
2 & 1980 & 288.0 & $20-22$ & useful plants, Araceae, Cactaceac - epiphytes \\
3 & 1980 & 288.0 & $10-15$ & succulents, Amaryllidaceae \\
4 & 1980 & 288.0 & $5-10$ & subtropical plants, Citrus, Fuchsia, Hihiscus \\
5 & 1992 & 288.0 & $18-22$ & tropical plants \\
6 & 1992 & 324.0 & $10-12$ & subtropical useful plants \\
A & 1981 & 21.6 & $20-22$ & tropical plants \\
B & 1981 & 43.2 & $10-12$ & Asparagus \\
C & 1981 & 43.2 & $5-10$ & no permanent collections \\
D & 1981 & 43.2 & 22 & Citrus, Coffea \\
E & 1981 & 86.4 & $5-10$ & no permanent collections \\
F & 1981 & 86.4 & - & no permanent collections \\
corridor (k) & $1980-1981$ & 258.0 & $8-10$ & Camelia Hedera \\
dendrological (x) & 1979 & 92.0 & & \\
\hline
\end{tabular}

The objects of this study were weeds in the broad sense, i.e. undesired plants, brought to the greenhouses either accidentally or deliberately, but self-perpetuating outside their plot without control. According to the definition by Zukowski (1993 - Słownik botaniczny, p. 93) weeds are native and introduced synanthropic plants occurring among cultivated plants in spite of human will and causing marked economic loss by qualitative and quantitative reduction of the vields [...]. This definition includes also garden-escapes. In the case of greenhouses, some exotic plants may be undesired, too, although they are not usually associated with the term weed. These species are called greenhouse-escapes here.

\section{MATERIAL AND METHODS}

Systematic checks repeated every two months were carried out in 1995, but earlier observations from 1992 and 1994 
were also taken into account. Floristic lists were made for each chamber separately.

Herbarium materials composed of 126 specimens of vascular plants and 35 samples of bryophytes were deposited in the Herbarium of the Botanical Garden in Powsin.

The scientific nomenclature follows that of Flora Europaea (Tutin et al. 1964, 1968, 1972, 1976, 1980), Zander Handwörterbuch der Pflanzennamen (Encke et al. 1994), Hortus Third (Bates et al. 1976) and Authors of Plant Names (Brummitt, Powell 1992). For some alien taxa other sources were used: Jones (1987) for ferns; Britton and Brown (1898) for Physalis spp.; and Jacobsen (1954) for Sedum and Euphorbia spp. The names of bryophytes used are those found in Ochyra and Szmajda (1992). Some taxa were represented by juvenile specimens only and it was sometimes impossible to identify them to species level, so they were determined at the genus level.

Data on the occurrence of plant species were analysed on the basis of various criteria. One of them was the origin and degree of naturalization of individual taxa. The distinguished elements of the flora were equivalents of geographical-historical groups according to Kornaś (1981), modified for the purposes of this study. The classification of life forms used is that of Raunkiaer (1934) and Zarzycki (1984). For the analyses only the basic categories were selected: megaphanerophytes, nanophanerophytes, chamaephytes, hemicryptophytes, cryptophytes and therophytes. In the case of exotic taxa which escaped from cultivation it was only noted whether the plant is an annual, a perennial, a shrub or a subshrub; data on their natural range were also recorded.

The list of taxa of vascular plants found in the greenhouses of the Botanical Garden in Powsin was compared with the register of spontaneously occurring species found outdoors in the area of this Botanical Garden (original research) and in greenhouses in Łódź (Wiśniewski, Asprou 1974).

\section{RESULTS}

\section{Floristic diversity}

In all the studied glasshouses a total of 243 taxa of vascular plants were found, including 216 taxa of species or lower rank and 4 hybrids (Table 2).

Out of the 67 plant families recorded in the greenhouses, 48 were found represented in the Polish flora (Table 2). The richest among them are: Asteraceae, Crassulaceae, Fabaceae and Polygonaceae. The following genera were represented by the highest number of taxa (i.e. by 5 taxa each): Begonia, Kalanchoe, Oxalis, Pilea, Rumex and Sedum.

Marked differences in the richness of the flora between the individual chambers of the greenhouses in Powsin were recorded (Table 4). The number of recorded taxa ranged from 20 to 90. The correlation between the richness of the flora of a given chamber and its age or area is not strong (Tables 1 and 4).

Among the 17 species of bryophytes found in the glasshouses, 14 were introduced accidentally, while the other 3 were used as a substrate for epiphytes (Table 3 ). The majority (16 species) were mosses, except for one liverwort, Marchantia polymorpha. Among them, Racopilum cuspidigerum and Taxiphyllum subarcuatum are new to the Polish flora (Rusinska et al. 1996). The others were cosmopolitan synanthropic taxa. Some, like Leptodictyum riparium, are associated with wet habitats. Two species typical of forests in general are also present: Brachythecium rutabulum and Plagiomnium cuspidatum. Bryophytes are most abundant in pots and crevices of the concrete floor. They also colonized the ground frequently.

\section{Elements of the studied flora}

The weeds of the greenhouses in Powsin are a very diverse group of plants. The division of the flora of the recorded vascular plants according to species origin and degree of naturalization is presented in the sections below. The flora includes both native species and aliens varying in the degree of naturalization (Table 2A-E). A separate group is composed of other taxa, for which detailed data are missing (Table 2F).

\section{Native plants}

Apophytes - here synanthropic species native to the Mazowsze region, were the most numerous group of weeds found in the area of Powsin greenhouses (Table 2A, Fig. 2). This group was composed of 89 taxa, which accounted for $36 \%$ of the studied flora. Frequency within this category was quite high - one apophytic species was found in 3.7 chambers on average. They dominated in the flora of all the chambers except Chamber 2 (Table 4), although the degree of their naturalization in the study area varied. Native weeds were most common in pots, near the planted specimens.

The dominant life form are perennials; among them hemicryptophytes rank first, as they are represented by 34 species, i.e. $39 \%$ of the native species (Fig. 3A). Annuals were also quite diverse ( 26 species, i.e. $29 \%$ of this group). Among the native species, virtually only therophytes were able to produce seed here. All the others were usually observed as either seedlings or juvenile specimens.

\section{Permanently established aliens}

Metaphytes were represented by 48 taxa, accounting for $20 \%$ of the studied flora (Table 2B, Fig. 2). This group included 29 archaeophytes, usually found in pots.

The number of permanently established aliens in individual chambers ranged from 3 to 30 taxa (Table 4). Their mean frequency was high - one taxon was found in 4.8 chambers on average. As far as life forms are concerned (Fig. 3B), plants characterized by short life cycles - therophytes - dominated among them (37 taxa, i.e. $77 \%$ of this group).

\section{Garden-escapes}

This group was represented by only 10 species $-4 \%$ of the flora of the greenhouses (Table 2C, Fig. 2). Single specimens were recorded in eight chambers (Table 4). They were cultivated exclusively in the ground, while spontaneously they occured in the greenhouses - mostly in pots. Each garden-escape was recorded in 1.5 chamber on average. They were mostly therophytes and hemicryptophytes (4 taxa each).

\section{Greenhouse-escapes}

This was a very diverse group of weeds -87 taxa, which accounted for $36 \%$ of the studied flora (Table 2D, Fig. 2). Comparison of the individual chambers with regard to the number of greenhouse-escapes (Table 4) showed that it ranged from 0 to 37. They were found mostly in patches of planted specimens and under the windowsills. They were most diverse in Chambers 1,3 and 5, and dominant in Chamber 2. One taxon of this group was found in 2.1 chambers on average. Most frequent were American and African species (27 and 19 taxa, respectively, see Table 2D). In the study area they were ergasiophygophytes. Among the life forms (Fig. 3C), perennials prevailed here -66 taxa (i.e. $76 \%$ greenhouse-escapes).

\section{Aliens introduced directly to greenhouses}

These were represented by two species: Ruellia caroliniensis and Pilea microphylla (Table 2E). They were perennials 
TABLE 2. List of the vascular plants in greenhouses in Botanical Garden in Powsin.

\section{A. Native taxa}

Name of taxon Family

Life-form

Chambers

)

* Achillea millefolium L.

* Acgopodium podagraria L.

* Anthriscus sylvestris (L.) Hoffm.

* Arabidopsis thaliana (L.) Meynh.

* Arenaria serpylliolia L.

* Artemisia vulgaris L.

* Athrium filix-femina (L.) Roth

* Atriplex hastata L.

* Berteroa incana (L.) DC

* Betula pendula Roth

Betula pubescens Ehrh.

Brachypodium sylvaticum (Huds.) P. Beauv.

* Cardamine hirsuta L.

* Cardaminopsis arenosa (L.) Hayck

* Carex sp.

* Carpinus hetulus L.

* Cerastium fontanum Baumg.

* Chelidonium majus L.

* Chenopodium alhum L.

* Chenopodium glaucum L.

Chenopodium polvspermum L.

* Chenopodium rubrum L.

* Cirsium arvense (L.) Scop.

* Dropteris carthusiana (Vill.) H.P.Fuchs

* Dryopteris filix-mas (L.) Schott

* Elirmus repens (L.) Gould

* Epilohium adenocaulon Hausskn.

* Epilohium hirsutum L.

Epilohium rescum Schreb.

- Equisetum arvense L.

* Erodium cicutarium (L.) L’Her.

* Erophila verma (L.) Chevall.

* Fesflica rubra L.

- Fragaria vesca $\mathrm{L}$.

* Galcopsis bifida Bocnn.

* Galium aparine L.

* Galium schultesii West

* Geraniam mohertianum L.

* Gnaphalium uliginosum L.

Hypericum humifusum L.

* Itypericum perforatum L.

* Juncus hufonius L.

* Juncus ceffusus L.

* Lapsana communis L.

* Lathvrus pratensis L.

* Lemina minor L.

* Linaria vulgaris Mill.

* Luzula campestris (L.) DC.

* Medicago lupulina L.

* Micelis muralis (L.) Dumort.

* Myosoton aquaticum (L.) Moench

* Plantago major L.

* Plantago major L. subsp. intermedia (DC.) Arcang.

- Plantago media $\mathrm{L}$

* Poa anmia L.

* Poa cfr. trivialis L.

* Polsgonatum odoratum (Mill.) Druce

* Poligonum aviculare L.

* Polygonam nodosum Pers.

* Polygomam persicaria L.

* Populus nigra L.

* Populas remula L.

* Potentilla collina Wibel

* Potentilla sp.

Quercus petraea (Matt.) Licbl.

* Quercus rohur L.

* Ranunculas ficaria L.

- Ranunculas repens $\mathrm{L}$.

* Ramenculas sceleratus L.

- Rorippa sylvestris (L.) Besser

* Rumer acetosa L.

- Rumer acetosella L.

* Rumex crispus L

- Rumer sp.

* Sagina procumbens L.

* Sambucus nigra L.

* Sedirm acre l.

Asteraceac

Apiaceac

Apiaceac

Brassicaceac
Caryophyllaceac

Asteraceac

Athyriaceac

Chenopodiaceac

Brassicaceac

Betulaceac

Betulaceac

Poaceac

Brassicaceac

Brassicaceac

Cyperaceae

Caryophyllaceac

Papaveraceac

Papaveraceac

Chenopodiaceac

Chenopodiaceac

Chenopodiaceac

Asteraceac

Aspidiaceac

Aspidiaceac

Poaceae

Onagraceac

Onagraceae

Onagraceac

Equisetaceac

Geraniaceac

Brassicaceae

Poaceac

Rosaceae

Lamiaceae

Rubiaceac

Rubiaceac

Geraniaceac

Asteraceac

Hypericaceae

Hypericaceac

Juncaceac

Juncaceae

Asteraceae

Fabaceac

Lemnaceae

Scrophulariaceac

Juncaceac

Fabaceac

Asteraceac

Caryophyllaceac

Plantaginaceac

Plantaginaceac

Plantaginaceae

Poaceac

Poaceac

Liliaceae

Polygonaceae

Polygonaceae

Polygonaceac

Salicaceae

Salicaccae

Rosaceac

Rosaceac

Fagaceac

Fagaceac

Ranunculaceac

Ranunculaceac

Ranunculaceac

Brassicaceac

Polygonaceae

Polygonaceac

Polygonaceae

Polygonaceac

Caryophyllaceac

Caprifoliaceac

Crassulaceac

Solanaceae

* Solamum dulcamara L.

* Sonchus arvensis L.

- Spergularia ruhra (L.) J.Presl el C.Presl

Asteraceae

Caryophyllaceac

Caryophyllaceae

Asteraceac

- Taraxacum officinale Weber

* Trifoliam arvense L.

* Trifolium repens L.

* Tussilago farfara L.

* Urtica dioica L.

Fabaceac

Fabaceac

Asteraceac

Urticaceac

Ericaceac

Vaccinium oxvenccos L.

* Verhascum sp.

* Veronica arvensis L.

* Veronica hederifolia L.

\begin{tabular}{|c|c|c|c|c|c|c|}
\hline $\mathrm{H}$ & k & + & & & & \\
\hline $\mathrm{H}$ & k & + & & & & \\
\hline $\mathrm{H}$ & B. $k$ & + & + & & & \\
\hline TH & I. $2,3,6, A, C, D, E, F, k$ & + & + & + & & \\
\hline $\mathrm{T}$ & k & + & & & & \\
\hline $\mathrm{Ch}$ & I. 2. 3.5.6, A. C. D. E, k & + & + & + & + & \\
\hline $\mathrm{H}$ & $5, \mathrm{~F}$ & + & & & & + \\
\hline $\mathrm{T}$ & 3 & & & + & & \\
\hline HT & k & & + & & & \\
\hline$M$ & 4. $6 . \mathrm{F}$ & + & + & & & \\
\hline M & 5 & & & & & + \\
\hline $\mathrm{H}$ & 4 & & + & & & \\
\hline $\mathrm{T}$ & I, 2, 3, 4, 5, A. C. E.F & + & + & + & & \\
\hline H & $\begin{array}{l}3,4,6 \\
5\end{array}$ & + & + & + & & + \\
\hline M & 3 & & & + & & \\
\hline $\mathrm{C}$ & $\mathrm{I}, 3,4,6, \mathrm{E}, \mathrm{k}$ & + & + & + & + & \\
\hline $\mathrm{H}$ & 1, 3, A, B, C, D. E, F, k, x & + & + & + & + & \\
\hline TH & $1,2,3,5,6, A, B, C, D, E, F, k, x$ & + & + & + & & \\
\hline $\mathrm{T}$ & 3 & & & + & & \\
\hline T & D & & & + & & \\
\hline $\mathrm{T}$ & 2 & & & + & & \\
\hline G & $3,4,5,6, C, D, E, F, k, x$ & + & + & + & + & \\
\hline HI & $\begin{array}{l}5 \\
5\end{array}$ & & & & & $\begin{array}{l}+ \\
+\end{array}$ \\
\hline G & 1. 3 & + & + & & & + \\
\hline
\end{tabular}

B. B. C. E, F, k, $x$

E, $k$

I. 2, E. k

$5,6, k$

I. 2, 3. B, E, k, $x$

,

1, 2,5

I, $3,4, \mathrm{k}$

I, 3, 5, C. D, E, $k$

I, 2, 3, 5, 6, B, C, k

3
5

I

1, 2, 3

2, E

2, 3, 4, A, D, F, k

1. 2. 3. 5, 6, A, B, C, D, E. F, k, x

F. $x$

4. B, C, k

2. 3, 4, A, C. D, E., k

A

I, 3, 4, 5, 6, C. k

1. $x$

4

B. $k$

I. 3, 4, 6, C, k

k

2. 3, 6, D

$2,3,4, \mathrm{~A}, \mathrm{C}, \mathrm{x}$

1. 2, 3, 6, C, E, F, k, $x$

$x$

A

C.

1, 5, 6, C, k

I. 2. 3, 5, A, B, C, D, E, F, k, $x$

I. 2. 3. 5, 6, A, B, C, D. E, F, k,

$1,3,4,5,6, B, k$

$1,3,6, \mathrm{~A}$

I, 2, 3, 5, 6, A, B, C, D. E. k, $x$

5

I, 2. 3. C. E

I, 2, 3, B, C, E,

I. 3. B. F, k, $x$

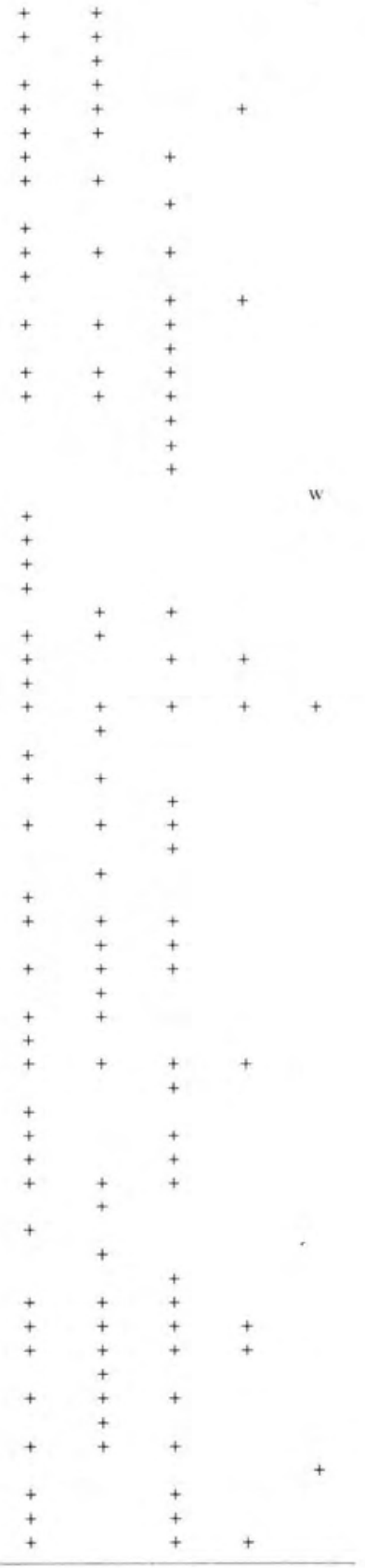


TABLE 2. Cont.

\begin{tabular}{|c|c|c|c|c|c|c|c|c|}
\hline \multicolumn{9}{|l|}{ B. Permanently established aliens } \\
\hline Name of taxon & Family & Life-form & Chambers & $\mathrm{P}$ & G & $\mathrm{O}$ & $\mathrm{s}$ & E \\
\hline * Amaranthus retroflexus L. & Amaranthaceae & $\mathrm{T}$ & 3.4. B. C & + & & + & & \\
\hline * Artemisia ahsinthium L. & Asteraceac & $\mathrm{Ch}$ & $\mathrm{k}$ & & & & + & \\
\hline${ }^{*}$ Atriplex nitens Schkuhr & Chenopodiaccac & $\mathrm{T}$ & k & & + & & & \\
\hline * Atriplex patula $\mathrm{L}$. & Chenopodiaceac & $\mathrm{T}$ & $6, A, k$ & + & + & & & \\
\hline * Bilderdikia convolvulus (L.) Dumon. & Polygonaccac & $\mathrm{T}$ & $3,5,6, \mathrm{E}, \mathrm{k}$ & + & + & + & & \\
\hline * Capsella hursa-pastoris (L.) Medik. & Brassicaceae & $\mathrm{T}$ & I, $2,5,6$, A. C, D. E, F, k & + & + & + & + & \\
\hline * Conza camadensis (L.) Cronquist & Asteraceac & $\mathrm{TH}$ & I. $2,3,5,6$, A. B, C. D. E, F, k & + & + & + & + & \\
\hline - Descarainia sophia (L.) Webb ex Prantl & Brassicaceac & $\mathrm{T}$ & 6 & & + & & & \\
\hline - Digitaria sanguinalis (L.) Scop. & Poaccac & $\mathrm{T}$ & $3, k$ & + & & + & & \\
\hline * Echinochloa crus-galli (L.) P.Beauv. & Poaceac & $\mathrm{T}$ & I, $2,3,5,6$, A, B, C. D, E, k & + & + & + & & \\
\hline * Euphorbia peplus L. & Euphorbiaceac & $\mathrm{T}$ & $5 . \mathrm{k}$ & + & + & & & \\
\hline * Galinsoga ciliata (Rafin.) S.F.Blake & Asteraceae & $\mathrm{T}$ & $2,3,5, \mathrm{~A}, \mathrm{~B}, \mathrm{C}, \mathrm{E}, \mathrm{k}, \mathrm{x}$ & + & + & + & & \\
\hline * Galinsoga parviflora Cav. & Asteraceac & $\mathrm{T}$ & $2,3,6, A, B, C, E, F, k$ & + & + & + & & \\
\hline * Geranium pesillum L. & Geraniaceac & $\mathrm{T}$ & I, 3, 5, 6, C, E, F, k & + & + & + & + & \\
\hline * Impatiens parviflora DC. & Balsaminaceac & $\mathrm{T}$ & $\mathrm{x}$ & + & & & & \\
\hline * Lactuca serriola L. & Asteraceac & $\mathrm{H}$ & I, 5. A. B. C. D. F. k & + & + & + & + & \\
\hline - Lamium amplexicaule L. & Lamiaceac & $\mathrm{T}$ & I. 2,3, B. E. k & + & + & + & & \\
\hline * Lamium purpureum L. & Lamiaceae & $\mathrm{TH}$ & I. $2,3,5,6$, B. C. D. E. $k$ & + & + & + & & \\
\hline - Malva neglecta Wallr. & Malvaceac & $\mathrm{HT}$ & $\mathrm{k}$ & + & + & & & \\
\hline * Matricaria maritima L. subsp. inodora (L.) Dostal & Asteraceac & TH & I, $2,3,4,5$, C. E. k & + & + & + & & \\
\hline * Mvosotis arvensis (L.) Hill & Boraginaceae & $\mathrm{TH}$ & i & & & + & & \\
\hline * Oenothera biennis L. & Onagraccac & $\mathrm{H}$ & $\mathrm{k}$ & + & & & & \\
\hline * Oenothera sp. & Onagraceac & & 1, $2,3,4,5, \mathrm{C}, \mathrm{k}$ & + & + & + & & \\
\hline * Oxalis cormiculata L. var. Corniculata & Oxalidaceac & $\mathrm{TH}$ & 1.4 & + & + & + & & \\
\hline * Oxalis corniculata L. var. repens (Thunb.) Zuce & Oxalidaceac & $\mathrm{TH}$ & I. $2,3,6, A, B, D, F, x$ & + & + & + & + & + \\
\hline Oxalis corniculata L. var. repens (Thunb.) Zuce f. purpurea Parl. & Oxalidaceac & $\mathrm{TH}$ & 3. F. k & + & & & + & \\
\hline * Oxalis stricta L. & Oxalidaceac & $\mathrm{T}$ & $1,2,3, A, B, C, E, F$ & + & + & + & + & \\
\hline Oxalis stricta L. x O. dillenii Jacq. & Oxalidaceac & $\mathrm{TH}$ & 3 & & + & & & \\
\hline * Papaver duthium L. & Papaveraceac & $\mathrm{T}$ & 4 & + & & & & \\
\hline * Polvgonum tomentosum Schrank & Polygonaceac & $\mathrm{T}$ & 3 & + & & & & \\
\hline * Quercus rubra L. & Fagaceac & M & I. F & & & + & & \\
\hline * Raphamus raphanistrum L. & Brassicaceac & $\mathrm{T}$ & 2. 6, B, k & + & + & + & & \\
\hline * Robinia pseudacacia L. & Fabaceae & M & 1. $2,5,6$, E. k & + & + & + & & \\
\hline * Rumex confertus Willd. & Polygonaceac & $\mathrm{H}$ & $\mathrm{F}$ & + & & & & \\
\hline * Senecio vernalis Waldst. et Kit. & Asteraceac & $\mathrm{T}$ & k & + & & & & \\
\hline * Senecio vulgaris L. & Asteraceae & $\mathrm{TH}$ & I. $2,3,6, A, C, D, E, F, k$ & + & + & + & + & \\
\hline * Setaria viridis (L.) P. Beauv. & Poaceac & $\mathrm{T}$ & 3 & + & & + & & \\
\hline * Sistmbrium sp. & Brassicaceac & & A & & + & & & \\
\hline * Solanum nigrum L. & Solanaceac & $\mathrm{T}$ & E & + & & & & \\
\hline * Solidago gigantea Aiton & Asteraccac & $\mathrm{HG}$ & 2 & + & & & & \\
\hline * Sonchus oleraceus L. & Asteraceac & $\mathrm{TH}$ & I. $2,3,5,6, A, B, C, k$ & + & + & + & + & \\
\hline * Spergula anensis L. & Caryophyllaceac & $\mathrm{T}$ & $3.6 . \mathrm{A}$ & + & + & + & & \\
\hline * Urtica urens L. & Urticaceae & $\mathrm{T}$ & 6, C, E. k & + & + & & & \\
\hline * Vicia hirsuta (L.) Gray & Fabaceac & $\mathrm{T}$ & 4. B. E & + & + & & & \\
\hline * Vicia sativa L. subsp. nigra (L.) Ehrh. & Fabaceae & $\mathrm{T}$ & I. $3.5, \mathrm{k}$ & + & + & + & - & \\
\hline * Vicia tetrasperma (L.) Schreb. & Fabaceac & $\mathrm{T}$ & I. $2,3,4,5,6, C, D, k$ & + & + & + & & \\
\hline * Viola arvensis Murray & Violaceac & $\mathrm{T}$ & $\mathrm{I}, 3,5,6, \mathrm{~B}, \mathrm{E}, \mathrm{k}$ & + & + & + & & \\
\hline
\end{tabular}

TABLE 2. Cont.

\begin{tabular}{|c|c|c|c|c|c|c|c|c|}
\hline \multicolumn{9}{|l|}{ C. Garden-escapes } \\
\hline Name of taxon & Family & Life-form & Chambers & $\mathrm{P}$ & $\mathrm{G}$ & 0 & $\mathrm{~s}$ & $\mathrm{E}$ \\
\hline * Amaranthus hrbridus L. & Amaranthaceac & $\mathrm{T}$ & 3 & & & + & & \\
\hline *Artemisia anmua $\mathrm{L}$. & Asteraceac & $\mathrm{T}$ & 3 & & & + & & \\
\hline * Calendula officinalis L. & Asteraceac & $\mathrm{T}$ & B & + & & & & \\
\hline Cotula squalida (Hook f.) Hook $f$. & Asteraceac & $\mathrm{H}$ & $\mathrm{x}$ & + & & & & \\
\hline * Datura sp. & Solanaceac & $\mathrm{T}$ & 2 & + & & & & \\
\hline * Galega officinalis L. & Fabaceae & $\mathrm{H}$ & $3,4,5,6$ & + & + & + & & \\
\hline * Lupinus sp. & Fabaccac & & $2,5.6$ & & + & + & & \\
\hline Samolus valcrandi $\mathrm{L}$. & Primulaceac & $\mathrm{H}$ & 4 & + & & & & \\
\hline * Sedum hispanicum L. & Crassulaceae & $\mathrm{H}$ & k & + & & & & \\
\hline Vinca major L. "Variegata" & Apocynaceac & $\mathrm{Ch}$ & B & & + & & & \\
\hline
\end{tabular}

that originated from the tropics, introduced with plant material imported directly to the Powsin greenhouses. They were recorded quite frequently (in 6.5 chambers on average), but only in the area of the complex of greenhouses (Table 4).

Flora of greenhouses vs. flora of the other parts of the Garden

In glasshouses a numerous group of vascular plants (147 species) was formed by taxa that occurred spontaneously in the Garden also outdoors. These plants accounted for $61 \%$ of the flora of greenhouse weeds. The taxa common to these two floras prevailed among the native plants (Table 2A), the permanently established aliens (Table 2B), and garden-escapes (Table 2C).

In this study a total of 96 taxa were found to occur spontaneously exclusively inside greenhouses ( $40 \%$ of the flora).
Neither during the original floristic studies that were carried out in 1991, nor in any earlier unpublished accounts (Bernat et al. 1955, Karcz 1980), these taxa were recorded in the Garden. This group was mostly composed of greenhouse-escapes that could be found exclusively in greenhouses. The only exception were four species that were cultivated both in the area of greenhouses and in other parts of the Garden (Table 2D).

\section{Most widespread taxa}

Among vascular plants, the most frequent weeds (15 taxa found in 10 or more chambers: Arabidopsis thaliana, Artemisia vulgaris, Capsella bursa-pastoris, Chelidonium majus, Chenopodium album, Cirsium arvense, Conyza canadensis, Echinochloa crus-galli, Epilobium sp., Lamium purpureum, 
TABLE 2. Cont.

\section{Greenhouse-escape}

\begin{tabular}{|c|c|c|c|c|c|c|c|c|c|}
\hline Name of taxon & Family & Life-form & Origin of taxon & Chambers & $\mathrm{P}$ & G & $\mathrm{O}$ & $\mathrm{S}$ & $\mathrm{E}$ \\
\hline Achimenes erecta (Lam.) H.P.Fuchs & Gesneriaceae & $\mathrm{p}$ & AM, sAN, nAS & $1,5, \mathrm{E}$ & + & + & + & + & + \\
\hline Achimenes sp. & Gesneriaceae & $\mathrm{p}$ & A & $1,2,3,5, k$ & & + & + & + & \\
\hline Adiantum capillus-veneris $\mathrm{L}$. & Adiantaceac & $\mathrm{p}$ & AM, nAS, sAN & $1,2,6, C, D, E$ & + & & + & + & \\
\hline Adiantum sp. & Adiantaceae & $\mathrm{p}$ & & I. $2,4,5, A, C, E, k$ & + & + & + & + & \\
\hline Adiantum tenerum Sw. & Adiantaceac & $\mathrm{p}$ & $\mathrm{AM}, \mathrm{nAS}$ & $\mathrm{E}$ & & + & & & \\
\hline Anthurium crassinervium (Jacq.) Schott & Araccae & $\mathrm{p}$ & sAS & 5 & & + & & & \\
\hline Aptenia cordifolia (L. f.) N.E.Br. & Aizoaceac & ss & sAf & $2,3,4$ & + & + & + & & \\
\hline Ardisia crenata Sims & Myrsinaceae & s & $\mathrm{meAz}$ & 2 & & & + & & \\
\hline Asparagus sp. & Liliaceac & $\mathrm{p}$ & & I, B & & + & & & \\
\hline Asplenium hulbiferum G.Forst. & Aspleniaceac & $p$ & Au. NZe, nind & 1 & & & + & & \\
\hline Begonia howerii Ziesenh. 'Nigra Magna' & Begoniaceae & $\mathrm{p}$ & hort & 2 & & + & & & \\
\hline Begonia bowerii Ziesenh. 'Tiger' & Begoniaceac & $\mathrm{p}$ & hort & 2 & + & + & & & \\
\hline Begonia me'allica W.G.Sm. & Begoniaceac & $\mathrm{p}$ & $\mathrm{AS}$ (Bras) & 5 & & + & & & \\
\hline Begonia sp. & Begoniaceac & $\mathrm{p}$ & & I. 2.4 .5 & + & + & + & & \\
\hline Begonia $\times$ hybrida hort. & Begoniaceae & $\mathrm{p}$ & hort & 1 & & + & & & \\
\hline Boussingaultia cordifolia Ten. & Basellaceac & $\mathrm{p}$ & meAS & 2, E, F & & + & & + & \\
\hline Bryonia alha $\mathrm{L}$. & Cucurbitaccae & $\mathrm{p}(\mathrm{H})$ & Eur, nAf, wAz & k & + & & & & \\
\hline Chirila lavendulacea Stapf & Gesneriaceae & $\mathrm{p}$ & $\mathrm{Az}(\mathrm{Mal})$ & 5 & & + & & & \\
\hline Chlorophy'um comosum (Thunb.) Jacques & Liliaceae & $\mathrm{p}$ & sAf & 2,3 & & & + & & \\
\hline Coffea arahica L. & Rubiaceac & s & tAf & $2,3,4, A . D$ & & + & & + & \\
\hline Crassula multicava Lem. & Crassulaceac & ss & sAf & 2. 3 & & + & + & & \\
\hline Crassula portulacea Lam. & Crassulaccac & s & sAf & 2. 3 & + & + & + & & \\
\hline Crassula sp. & Crassulaceac & & & 3 & & + & + & & \\
\hline Cyclosorus dentatus (Forssk.) Ching & Thelypteridaceac & $\mathrm{p}$ & pant & I. $2,4,5,6, D, E, F, k$ & + & + & + & + & + \\
\hline Cyperus sp. & Cyperaceac & $\mathrm{p}$ & & I. 2, 3, 6, C.E & + & + & + & + & + \\
\hline Diescorca sp. & Discoreaceac & $\mathrm{p}$ & & $1,2,5, \mathrm{k}$ & + & + & + & + & \\
\hline Doodia sp. & Blechnaceac & $\mathrm{p}$ & & 5 & & + & & & \\
\hline Dorstenia sp. & Moraceac & $\mathrm{p}$ & & 5 & & + & & & \\
\hline Euphortia huhalina Boiss. & Euphorbiaceac & s & sAf & 3 & & + & & & \\
\hline Euphorbia characias L. & Euphorbiaceac & $\mathrm{p}$ & eMed & 3 & & & + & & \\
\hline Euphorbia sp. & Euphorbiaceac & & & 3 & & + & & & \\
\hline Fuchsia arborescens Sims & Onagraceae & s & $\mathrm{AM}$ & 5 & & + & & & \\
\hline Fuchsia procumbens R.Cunn. ex A.Cunn. & Onagraceae & s & $\mathrm{Au}$ & 3. A & + & & + & & \\
\hline $\begin{array}{l}\text { * Gladiolus efr. x gandavensis Van Houtte } \\
\text { Giraptopetalum paraguavense (N.E.Br.) E. Walther }\end{array}$ & Iridaceac & $\mathrm{p}(\mathrm{G})$ & hort. & 1. 4. D, E, k & + & + & + & & \\
\hline $\begin{array}{l}\text { Graptopetalum paraguavense (N.E.Br.) E. Walther } \\
\text { Hipoestes phy/lostachra Baker }\end{array}$ & Crassulaceac & $p$ & AM? & 2 & & & & + & \\
\hline $\begin{array}{l}\text { Hypoestes phillostachia Baker } \\
\text { Impatiens balsamina } \mathrm{L} \text {. }\end{array}$ & Acanthaceac & $s$ & Af(Madag) & 1. 2.5 & $\begin{array}{l}+ \\
+\end{array}$ & + & & & \\
\hline $\begin{array}{l}\text { Impatiens balsamina L. } \\
\text { Impatiens walleriana Hook.f. }\end{array}$ & Balsaminaceac & a & $\mathrm{SAz}$ & $\begin{array}{l}\mathrm{E} \\
2 . \mathrm{E}\end{array}$ & + & + & & + & \\
\hline $\begin{array}{l}\text { Impatiens walleriana Hook.f. } \\
\text { Kalanchoce hlossfeldiana Poelln. }\end{array}$ & $\begin{array}{l}\text { Balsaminaccac } \\
\text { Crassulaceac }\end{array}$ & p ss & $\begin{array}{l}\text { teAf } \\
\text { Af(Madag) }\end{array}$ & $\begin{array}{l}\text { 2. E } \\
2 . \mathrm{k}\end{array}$ & + & 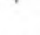 & + & & \\
\hline $\begin{array}{l}\text { Kalanchoe hlossfeldiana Poelln. } \\
\text { Kalanchoe daigremontiana Raym.-Hamet et H.Perrier }\end{array}$ & $\begin{array}{l}\text { Crassulaccae } \\
\text { Crassulaceac }\end{array}$ & ss & Af(Madag) & 3 & 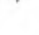 & + & + & + & \\
\hline Kalanchoe rotundifolia (Haw.) Haw. & Crassulaceac & $\mathrm{p}$ & sAf & 3 & & & + & + & \\
\hline Kalanchoe sp. & Crassulaceac & $\mathrm{p}$ & Af? & 3 & & + & + & & \\
\hline Kalanchoe tuhiflora (Harv.) Raym.-Hamet & Crassulaceac & $\mathrm{p}$ & Af(Madag) & 2. 3, C & & + & + & + & \\
\hline * Licopersicon esculentum Miller & Solanaceac & a & wAS & 4 & & + & & & \\
\hline Macrothelypteris sp. & Thelypteridaceae & $\mathrm{p}$ & & 2, E & & + & & & \\
\hline Nephrolepis exaltata (L.) Schott & Nephrolepidiaceac & $\mathrm{p}$ & sAN, AM, nAS & C & & + & & + & \\
\hline Origanum majorana $\mathrm{L}$. & Lamiaceac & a & nAf, swAz & C & & + & & & \\
\hline Passiflora cacrulea L. & Passifloraceac & s & seAS & 4 & & + & & & \\
\hline Passiflora edulis Sims & Passifloraceac & s & $\mathrm{A}($ Bras $)$ & E & & + & & & \\
\hline Passiflora sp. & Passifloraceac & s & A? & $1,4, F$ & & + & & & \\
\hline Passiflora violacea Vell. & Passifloraceac & s & mAS & 1 & & + & & + & \\
\hline Peperomia caperata Y unck. & Piperaceae & $\mathrm{p}$ & $\mathrm{AS}$ (Bras?) & 5 & & + & & & \\
\hline * Physalis philadelphica Lam. & Solanaceae & a & AS & k & + & & & & \\
\hline Pilea depressa (Schwartz) Blume & Urticaceac & $\mathrm{p}$ & Az(wind) & $\mathrm{E}$ & & + & & + & \\
\hline Pilea repens (Sw.) Wedd. & Urticaceac & $\mathrm{p}$ & $\mathrm{AM}$ & 4 & & & + & & \\
\hline Pilea sp. & Urticaceac & $\mathrm{p}$ & A & 2,5 & & + & & + & + \\
\hline Pilea spruceana Wedd. & Urticaceae & $\mathrm{p}$ & WmAS & 2 & & + & & & \\
\hline Polyxtichum falcatum (L.f.) Diels & Aspidiaceac & $\mathrm{p}$ & Az, sAf & $2,3,5,6, D, E, F, k$ & + & + & & + & \\
\hline Psilotum mudum (L.) P. Beauv. & Psilotaceac & $\mathrm{p}$ & pant & 2,5 & & + & & & \\
\hline Pteris cretica L. & Pteridaceac & $\mathrm{p}$ & t. subt & 5. A. D. E. k & + & + & & + & \\
\hline Pteris creticu L. 'Major' & Pteridaceae & $\mathrm{p}$ & hort & I. D. k & & + & + & & \\
\hline Pteris cretica L. 'Wilmsetti" & Ptcridaceac & $\mathrm{p}$ & hort & $\mathrm{E}$ & & + & & & \\
\hline Pleris sp. & Pteridaceac & $\mathrm{p}$ & & 5. E & + & + & & + & \\
\hline Pteris tremula R.Br. & Pteridaceac & $\mathrm{p}$ & $\mathrm{Au}$ & 1, 5, D & & + & + & & \\
\hline Pteris vittata $\mathrm{L}$. & Pteridaceae & $\mathrm{p}$ & tAz. $\mathrm{tAf}^{\circ}$ & 2. 5. D. E. k & + & + & + & + & \\
\hline Saintpaulia ionantha H.Wendl. & Gesneriaceac & $\mathrm{p}$ & sAf & 1,2 & & + & + & & \\
\hline Saxifraga sarmentosa L.f. & Saxifragaceac & $\mathrm{p}$ & $\mathrm{eAz}$ & I & & + & & & \\
\hline Scadoxus multiflorus (Martyn) Raf. & Amaryllidaceae & $\mathrm{p}$ & esAf & 2 & & + & & & \\
\hline Sedum dasyphy/lum L. & Crassulaceac & $\mathrm{p}$ & sFur. nAf & 3 & & & + & & \\
\hline Sedum sp. & Crassulaceac & $\mathrm{p}$ & & 3 & & & + & & \\
\hline Sedum stahlii Solms & Crassulaceac & $\mathrm{p}$ & $\mathrm{A}(\mathrm{Mex})$ & 3 & & + & + & & \\
\hline Selaginella sp. & Selaginellaceac & $\mathrm{p}$ & Az? & 1. $2, \mathrm{k}$ & + & + & + & + & \\
\hline Sinocrassula indica (Decne.) A.Berger & Crassulaceae & $\mathrm{p}$ & $\mathrm{sAz}$ & 2.3 & & + & + & & \\
\hline Soleirolia soleirolii (Req.) Dandy & Urticaceac & $\mathrm{p}$ & sEur. & 2. 4 & & + & + & + & \\
\hline Streptocarpus grandis N.E.Br. ex C.B.Clarke & Gesneriaceac & $\mathrm{p}$ & sAf & 5 & & + & & & \\
\hline Streptecarpus rexii (Bowic ex Hook.) Lindl. & Gesneriaceac & $\mathrm{p}$ & sAf & C & & + & & + & \\
\hline Sircptocarpus sp. & Gesneriaceac & $\mathrm{p}$ & & $1, k$ & + & & + & & \\
\hline Streptocarpus x hrbridus Voss. & Gesneriaceac & $\mathrm{p}$ & hort & 1 & & & + & & \\
\hline Singonium podophy/lum Schott & Araceac & s & $\mathrm{AM}$ & 2 & & + & & & \\
\hline Tectaria cicutaria (L.) Copel. & Aspidiaccac & $\mathrm{p}$ & pant & $\mathrm{C}$ & & + & & & \\
\hline Tetranema roseum (M.Martens et Galcotti) Standl. ct Steyerm. & Scrophulariaccac & $\mathrm{p}$ & $\mathrm{AM}$ & 5 & & + & & & \\
\hline Tradescantia sp. & Commelinaceae & $\mathrm{p}$ & A & 1. 2 & & + & + & + & \\
\hline Veltheimia sp. & Liliaceac & $\mathrm{p}$ & sAf & 4 & & + & & & \\
\hline Woodwardia orientalis Sw. & Blechnaceac & $\mathrm{p}$ & $\mathrm{Az}$ & 6 & & & & + & \\
\hline Zehrina pendula Schinzl. & Commelinaceac & $\mathrm{p}$ & $\mathrm{A}(\mathrm{Mex})$ & 2 & & + & & & \\
\hline Zehrina purpusii G. Bruckn. & Commelinaceae & $\mathrm{p}$ & $A(M e x)$ & 1,2 & & + & & & \\
\hline Zehrina sp. & Commelinaceac & $\mathrm{p}$ & $\mathrm{A}$ & 2,3 & + & + & & + & \\
\hline
\end{tabular}


TABLE 2. Cont.

\section{E. Aliens introduced directly to greenhouses}

\begin{tabular}{|c|c|c|c|c|c|c|c|c|c|}
\hline Name of taxon & Family & Life-form & Origin of taxon & Chambers & $\mathrm{P}$ & G & $\mathrm{O}$ & $\mathrm{S}$ & $\mathrm{E}$ \\
\hline Pilea microphylla (L.) Liebm & Iridaceae & $\mathrm{p}$ & $\mathrm{tA}$ & $1,4,5, \mathrm{~A}, \mathrm{E}, \mathrm{k}$ & + & + & + & + & \\
\hline Ruellia caroliniensis (J.F.Gmel) Steud & Urticaceae & $\mathrm{p}$ & SAN, AM & $1,2,5, A, B, C, E$ & + & + & + & + & \\
\hline
\end{tabular}

TABLE 2. Cont.

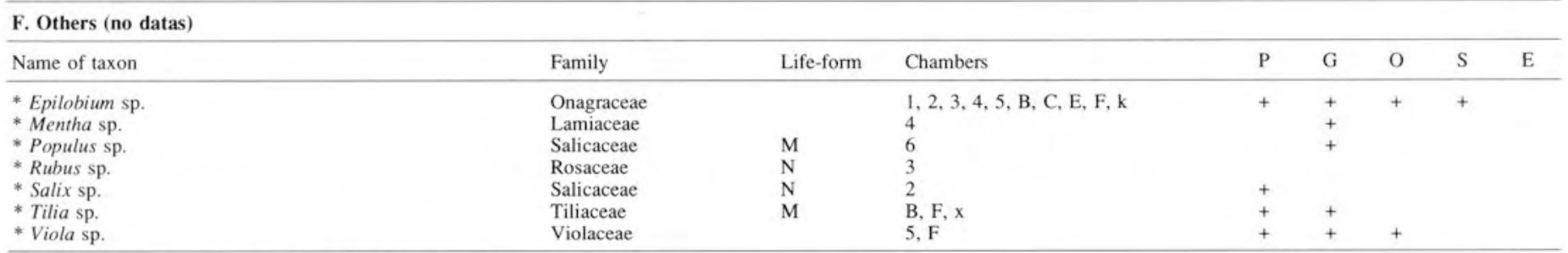

Explanatory notes for Tables $2 \mathrm{~A}, 2 \mathrm{~B}, 2 \mathrm{C}, 2 \mathrm{~F}$

* - taxa common with greenhouses and other part of Garden; Life-form: M - megaphanerophytes, N - nanophanerophytes, H - hemicryptophytes, Ch chamaephytes, C - cryptophytes, G - geophytes, Hy - hydrophytes, T - therophytes; Chambers - occurence in each chambers; P - pots, G - patches (i.e. in the ground), $\mathrm{O}$ - windowsills, $\mathrm{S}$ - slots in concrete, $\mathrm{E}$ - supports for epiphytes, w - water body. Abbreviations consistent with Table 1.

Explanatory notes for Tables 2D, $2 \mathrm{E}$

* - taxa common with greenhouses and other part of Garden; Life-form: s - shrub, ss - semishrub, p - perennial plant, a - annual plant; Origin of taxon: e - eastern, $\mathrm{n}$ - northen, s - southern, $\mathrm{w}$ - western, $\mathrm{m}$ - central, $\mathrm{t}$ - tropical, pant - pantropical, subt - subtropical, hort - horticultural; A America, Af - Africa, AM - Central America, AN - Northen America, AS - Southen America, Au - Australia, Az - Asia, Bras - Brazil, Eur - Europe, Ind - India, Madag - Madagascar, Mal - Malaya Archipelago, Med - Mediterranean Area, Mex - Mexico, Nze - New Zealand; Chambers - occurence in each chambers; $\mathrm{P}$ - pots, $\mathrm{G}$ - patches (i.e. in the ground), $\mathrm{O}$ - windowsills, $\mathrm{S}$ - slots in concrete, $\mathrm{E}$ - supports for epiphytes. Abbreviations consistent with Table 1

Poa annua, Senecio vulgaris, Stellaria media, Taraxacum officinale, Urtica dioica) were species that were also found outdoors, in other parts of the Garden. The majority (9) were species with short life cycles - therophytes (see Table 2). The geographical-historical classification showed that most numerous among them were native taxa.

Among greenhouse-escapes most common were ferns: $\mathrm{Cy}$ closorus dentatus, Polystichum falcatum and Adiantum spp. (Table 2D). The most frequent bryophytes were Leptodictyum riparium and Amblystegium serpens.

\section{DISCUSSION}

In greenhouses covering $0.6 \%$ of the area of the Botanical Garden in Powsin, 243 taxa of spontaneously occurring vascular plants were recorded, while in the other parts of the

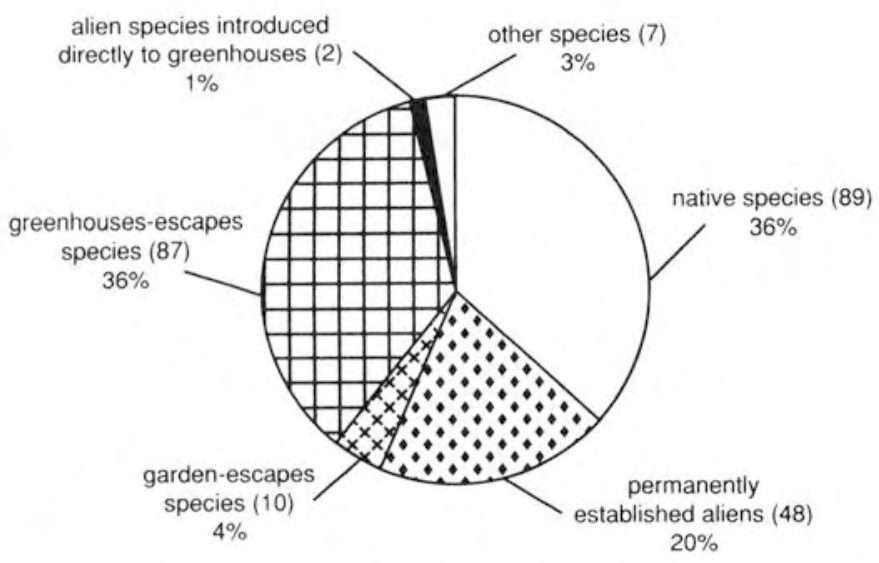

Fig. 2. Elements of the studied flora according to species origin and degree of naturalization (in brackets number of taxa).
TABLE 3. List of Bryophyta in greenhouses in Botanical Garden in Powsin.

\begin{tabular}{l|l}
\hline Name of taxon & Chambers \\
\hline - Aulacomnium palustre (Hedw.) Schwaegr. & 5 \\
- Pohlia nutans (Hedw.) Lindb. & 5 \\
- Sphagnum sp. & 5 \\
Amblystegium serpens (Hedw.) B., S. \& G. & $1,2,3,4, \mathrm{~A}, \mathrm{~B}, \mathrm{D}, \mathrm{k}$ \\
Brachythecium rutabulum (Hedw.) B., S. ZG. & $4, \mathrm{C}$ \\
Bryum argenteum $\mathrm{Hedw}$. & $1,3, \mathrm{x}$ \\
Bryum capillare Hedw. & $\mathrm{x}$ \\
Ceratodon purpureus (Hedw.) Brid. & $4,5, \mathrm{~B}, \mathrm{k}, \mathrm{x}$ \\
Funaria hygrometrica $\mathrm{Hedw}$. & $4,5, \mathrm{~F}, \mathrm{k}, \mathrm{x}$ \\
Leptodictyum riparium (Hedw.) Warnst. & $1,2, \mathrm{~A}, \mathrm{~B}, \mathrm{C}, \mathrm{D}, \mathrm{E}, \mathrm{k}, \mathrm{x}$ \\
Letobryum pyriforme (Hedw.) Wils. & $1,4,5,6, \mathrm{~F}, \mathrm{k}, \mathrm{x}$ \\
Marchantia polymorpha L. & $\mathrm{F}, \mathrm{x}$ \\
Plagiomnium cuspidatum (Hedw.) T. Kop. & $\mathrm{D}$ \\
Racopilum cuspidigerum (Schwaegr.) Angstr. & $5, \mathrm{E}$ \\
Steblotrichum convolutum (Hedw.) P. Beauv. & $1,3,4,5,6, \mathrm{~B}$ \\
Taxiphyllum subarcuatum (Broth.) Iwats & $\mathrm{E}$ \\
Tortula muralis Hedw. & $\mathrm{x}$ \\
\hline
\end{tabular}

- species which make breeding-ground for epiphytes, Chambers shortenings consistents with Table 1

Garden 450 species were found (original research). Considering the marked differences in the size of these areas it may be concluded that the Powsin greenhouses are characterized by a very rich flora.

The most numerous group is formed by taxa native to the Mazowsze region (37\% of the studied flora). The importance of this element is also attested to by its dominance in the flora of nearly all the greenhouse chambers, the relatively high frequency and among the weeds that were recorded most frequently. The majority of the apophytes are hemicryptophytes and therophytes.

The native flora is relatively poor $(36 \%)$ while permanently established aliens constitute $20 \%$ of the studied flora. Ergasio- 


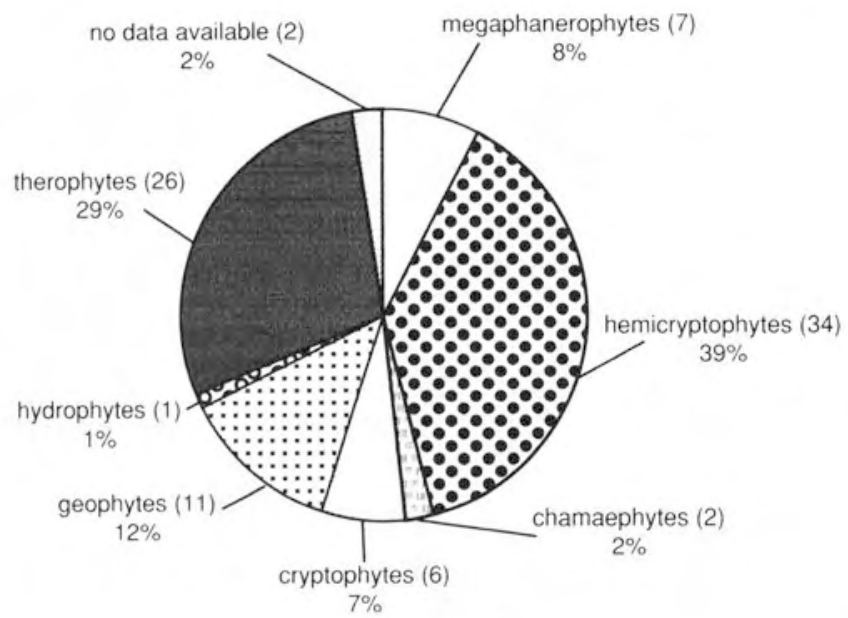

Fig. 3A. Life-form spectrum of native species (in brackets number of taxa).

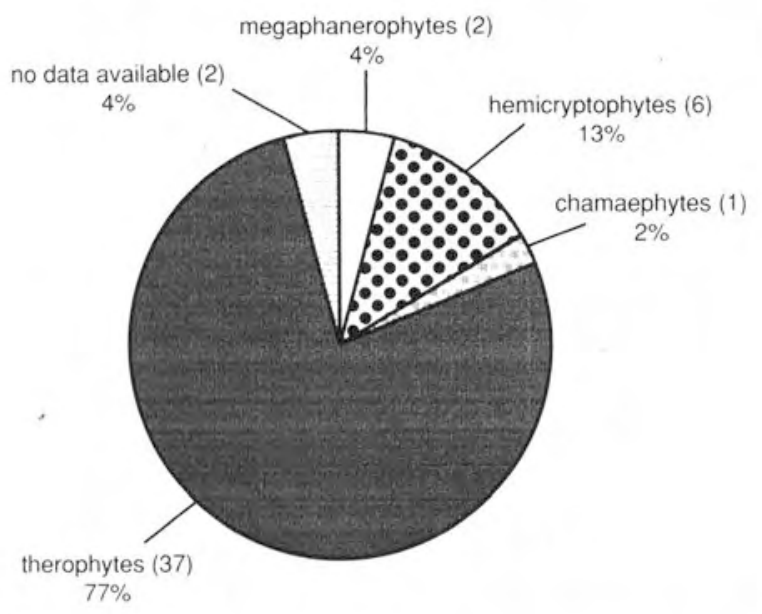

Fig. 3B. Life-form spectrum of permanently established aliens (in brackets number of taxa).

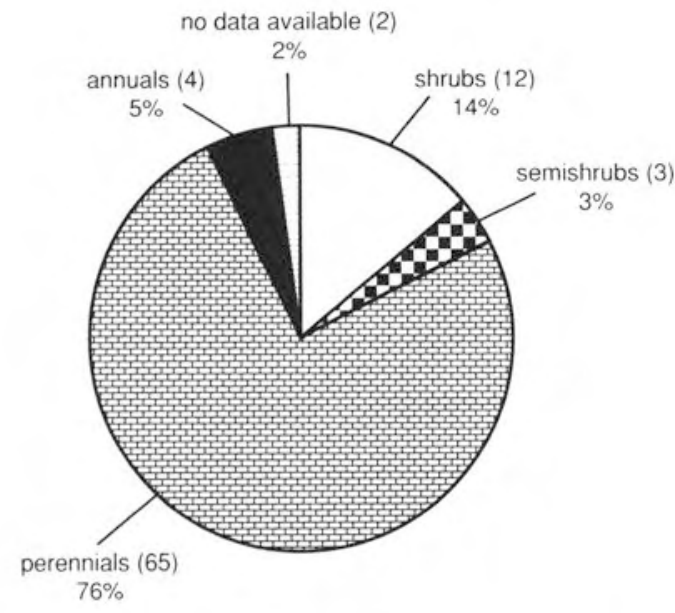

Fig. 3C. Life-form spectrum of greenhouse-escapes (in brackets number of taxa).

phygophytes i.e. greenhouse-escapes and garden-escapes constitued as much as $40 \%$ of the flora.

A comparative analysis of the above data with the results obtained by Sudnik-Wójcikowska (1987) suggests that the flora of Powsin glasshouses was dominated by diaphytes ( $40 \%$ of the flora), while their proportion the present-day sy- nanthropic flora of Warsaw is only $20 \%$. In the case of apophytes and metaphytes the opposite tendencies are the observed: in the area of Warsaw they account for $56 \%$ and $24 \%$ of the flora, respectively, while in the flora of the greenhouses their proportion is much smaller (37 and 19\%).

Observations made in the areas adjacent to the greenhouses allowed for investigation of the interaction between the spontaneous flora of the greenhouses and the plants growing in their immediate neighbourhood. The flora of greenhouse weeds was dominated by species that occured spontaneously also in other parts of the Garden, and it may be assumed that most of them derive from the plants growing nearby. A total of 147 taxa were recorded in this group, which accounts for $61 \%$ of the greenhouse flora. Most of them were apophytes, and metaphytes.

The taxa occurring spontaneously in the area of the greenhouses were mostly greenhouse-escapes. They formed a group of ergasiophygophytes specific to the study area and lacking the tendency to colonize the areas around the glasshouses. These were mostly perennials. This results mostly from the fact that selection of exotic species for expositions - about $70 \%$ of them are perennials.

Another 13 species found exclusively inside the greenhouses were of various origin. Two of them were cultivated only in the ground outdoors: Cotula squalida and Samolus valerandii. The former was propagated vegetatively, but it has spontaneously spreading specimens found in the small dendrological greenhouse, which is about $40 \mathrm{~m}$ away from the place where it is cultivated. The latter has migrated to the complex of greenhouses from a patch $30 \mathrm{~m}$ away. Two other species (Ruellia caroliniensis and Pilea microphylla) come from the tropics, and have been introduced accidentally to the greenhouses, with imported plant materials. Seeds of Betula pubescens and Vaccinium oxycoccos, which are native to the Mazowsze region, have certainly been brought to the greenhouses with the substrate which is used as a covering for branches that support epiphytes. The origin of the other seven taxa found only indoors (Brachypodium sylvaticum, Chenopodium polyspermum, Hypericum humifusum, Epilobium roseum, Quercus petraea, Oxalis corniculata var. repens f. purpurea and $O$. stricta $\times O$. corniculata) is not certain, but it is highly probable that their diaspores have been brought here with substrates.

During the regular and detailed floristic observations made in the Botanical Garden in Powsin no case of expansion of exotic plants into the areas outside the greenhouses was recorded, although literature data suggest that this is possible. For example, spontaneous occurrence of three weeds that probably originated from exposition greenhouse were recorded in the Botanical Garden of the Jagiellonian University in Kraków: Chenopodium giganteum Don., Nicandra physaloides (L.) Gaertn. and Oxalis corniculata L. (Trzcińska-Tacik 1971, 1979). The latter species was also recorded in the Moscow Botanical Garden (Kozhevnikov 1935): it was very common in greenhouses, from where it migrated to flower beds outside.

The flora of vascular plants of the greenhouses of the Botanical Garden in Powsin is very rich and varied in comparison with the greenhouse flora studied in the city of Łódź (Wiśniewski, Asprou 1974). In the Powsin glasshouses that cover nearly $2.500 \mathrm{~m}^{2}, 243$ taxa were recorded, while in Łódź only 72 species were found to occur spontaneously in seven productive greenhouses (a total of $168000 \mathrm{~m}^{2}$ ). These differences result mostly from the types of plants grown there: in Łódź only vegetables and ornamental plants were cultivated, 
TABLE 4. Number of vascular plants in each chamber - shortenings consistents with Table 1.

\begin{tabular}{|c|c|c|c|c|c|c|c|c|c|c|c|c|c|c|c|}
\hline \multirow[t]{2}{*}{ Group of taxa } & \multicolumn{14}{|c|}{ Chambers } & \multirow[t]{2}{*}{ Total } \\
\hline & 1 & 2 & 3 & 4 & 5 & 6 & A & B & $\mathrm{C}$ & D & E & $\mathrm{F}$ & k & $\mathrm{x}$ & \\
\hline Native taxa & 30 & 25 & 39 & 15 & 25 & 22 & 15 & 19 & 25 & 15 & 21 & 15 & 39 & 15 & 90 \\
\hline Permanently established aliens & 20 & 17 & 24 & 7 & 16 & 18 & 13 & 14 & 16 & 8 & 17 & 11 & 29 & 3 & 47 \\
\hline Garden-escapes & 0 & 2 & 3 & 2 & 2 & 2 & 0 & 2 & 0 & 0 & 0 & 0 & 1 & 1 & 10 \\
\hline Greenhouses-escapes & 24 & 37 & 22 & 12 & 23 & 5 & 4 & 1 & 9 & 9 & 18 & 4 & 12 & 0 & 87 \\
\hline Aliens introduced directly to greenhouses & 2 & 1 & 0 & 1 & 2 & 0 & 2 & 1 & 1 & 0 & 2 & 0 & 1 & 0 & 2 \\
\hline Others & 1 & 2 & 2 & 2 & 2 & 1 & 0 & 2 & 1 & 0 & 1 & 3 & 1 & 1 & 7 \\
\hline Total & 77 & 84 & 90 & 39 & 70 & 48 & 34 & 39 & 52 & 32 & 59 & 33 & 85 & 20 & 243 \\
\hline
\end{tabular}

so the weeds were mostly segetal species. According to above authores these are mostly mesophytes.

One of the most persistent greenhouse weeds, both in Łódź and in Powsin, was Poa annua. In Powsin it was found in nearly all the chambers. In the glasshouses in Łódź Stellaria media, Galinsoga ciliata and Juncus bufonius were also very frequent, while in Powsin Chenopodium album and Taraxacum officinale were found in the highest number of chambers.

The floristic diversity does not seem to be affected by glasshouse size. This is confirmed by the comparison of results obtained in Powsin and Łódź (Wiśniewski, Asprou 1974), and of the number of taxa recorded in individual chambers of the Powsin greenhouses. The types of plants cultivated there are of much greater importance.

The mode of greenhouse use is also directly related to the number and combination of vascular plant families found in the study area. Among the 67 plant families recorded in the Powsin glasshouses, 19 have no representatives in the Polish flora. In Łódź (24 families) no exotic species were found. The most numerous members of the Asteraceae dominated also the flora of Łódź greenhouses, and the flora of Warsaw and Poland (Sudnik-Wójcikowska 1987). By contrast, the family Crassulaceae, which ranked second in the study area, has few representatives in the Polish flora, while Wiśniewski and Asprou (1974) recorded no members of this family in Łódź.

Among the 17 species of bryophytes found in the greenhouses of the Botanical Garden in Powsin, 14 may be regarded as introduced accidentally, while the other three as introduced deliberately as a substrate for epiphytes.

\section{CONCLUSIONS}

The flora of greenhouses is a very interesting, although poorly studied subject of research. The results, apart from the cognitive aspect, are also of practical importance because some of the plants occurring spontaneously in greenhouses are persistent weeds.

The spontaneous flora of the glasshouses of the Botanical Garden in Powsin is very rich and diversified. It includes 17 species of bryophytes and 243 taxa of vascular plants. The flora is dominated by plants growing also outdoors in the immediate neighbourhood, i.e. in other parts of the Garden. The second largest group are greenhouse-escapes.

The interaction between the weed flora of greenhouses and the plants occurring in the vicinity of the glasshouses is unilateral. No cases of expansion of greenhouse plants into other parts of the Garden have been recorded, although colonization of greenhouses by outdoor plants is very common.
On the basis of observations carried out in the area of the Garden 4 potential sources of diaspores for plants noted in greenhouses can be distinguished. These include:

- plants occurring spontaneously in the area of the Garden,

- plants cultivated in the Garden,

- plants grown exclusively in the greenhouses,

- plants introduced directly to the greenhouses from other sources.

The significance of the individual sources is difficult to evaluate, although the flora of other parts of the Garden seems to be most important. Plants grown in the greenhouses rank second. Some of them have a capacity for intensive spontaneous dispersal, but do not show any tendency to expand outdoors.

The mode of greenhouse use affecting the environmental conditions and sources of diaspores, has a decisive influence on the specificity of the studied flora.

\section{ACKNOWLEDGEMENTS}

We would like to give sincere thanks to the wardens of the greenhouse collections of the Botanical Garden - Centre for Biodiversity Preservation in Powsin, especially to $\mathrm{Mr}$ Wiesław Gawryś for helping us to identify some exotic species. We wish to express our appreciation to Dr Hanna Rusińska for identification of bryophytes and their description, and to Dr Jacek Hantz for identification of taxa of the genus Oxalis. Our sincere thanks for valuable advice and discussion of the results of this study are due to Prof. Karol Latowski, Dr Bogdan Jackowiak and Dr Barbara Sudnik-Wójcikowska. We are also very grateful to our colleagues, Mr Maciej Niedzielski and Dr Wojciech Szwed, who have helped us to apply numerical methods.

\section{LITERATURE CITED}

BATES D.M., INGRAM J.W., MOORE H.E. (eds.). 1976. Hortus Third. A Concise Dictionary of Plants Cultivated in the United States and Canada. Macmillan Publishing Company, Collier Macmillan Publishers, New York, London.

BERDOWSKI W. 1988. Flora mchów Ogrodu Botanicznego we Wroclawiu. Acta Univ. Wratisl., Pr. Bot. 40: 47-59.

BERNAT J., PODBIELKOWSKI Z., ŻYŁKA R. 1955. Zbiorowiska roślinne terenów Centralnego Ogrodu Botanicznego PAN w Powsinie k. Warszawy. Botanical Garden of the Polish Academy of Sciences (mscr.).

BRITTON N., BROWN A. 1898. Illustrated Flora of the Northern United States, Canada and the British Possessions. Charles Scribner's Sons, New York, v. 3. 
BRUMMITT R.K., POWELL C.E. (eds.). 1992. Authors of plant names. Whitstable Litho Ltd., Whistable.

ENCKE F., BUCHHEIM G., SEYBOLD S. 1994. Zander Handwörterbuch der Pflanzennamen, 15. Auflage. Verl. E.Ulmer, Stuttgart.

FILIPIAK E. 1996. Brioflora Ogrodu Botanicznego w Łodzi. In: Kurzac T. (ed.). Przyroda Ogrodu Botanicznego w Łodzi. Oficyna Wydawniczo-Reklamowa "Sagalara", Łódź. pp. 91-96.

JACOBSEN H. 1970. Das Sukkulentenlexicon. VEB Gustav Fischer Verl., Jena.

JONES D.L. 1987. Encyclopedia of Ferns. An Introduction to Ferns, their Structure, Biology, Economic Importance, Cultivation and Propagation. Timber Press, Portland.

KARCZ E. 1980. Inwentaryzacja flory terenu I etapu budowy OB PAN oraz 40 hektarów przyległego lasu. Botanical Garden of the Polish Academy of Sciences (mscr.).

KORNAŚ J. 1981. Oddziaływanie człowieka na florę: mechanizmy i konsekwencje. Wiad. Bot. 25.3: 165-182.

KOZHEVNIKOV A.V. 1935. Sornaja i adventivnaja flora Moskovskogo botanicheskogo sada. Bjulleten Moskovskogo Obščestva Ispytatelej Prirody, Otd. Biol. 4: 193-203.

MENZEL M. 1984. Die Moosflora des Botanischen Gartens Berlin-Dahlem. Verhandl. des Berl. Bot. Ver. 3: 25-62.

OCHYRA R., SZMAJDA P. (eds.). 1992. Atlas of the geographical distribution of mosses in Poland. Part 8. W. Szafer Institute of Botany of the Polish Academy of Sciences, Adam Mickiewicz Uniwersity, Kraków, Poznań.

PILOUS Z. 1956. Fragmenta bryologica 2. Tropický mech z Lednice. Preslia 28: 43.

PODPERA J. 1949a. Zajimovy tropický mech v nasich sklenicich (Rhacopilum cuspidigenum Schwaegr.). Ceskoslov. Bot. Listy 2.1: 1-3, 16.

PODPERA J. 1949b. Zajimovy tropický mech v nasich sklenicich. Ceskoslov. Bot. Listy 2.2-3: 27.

RAUNKIAER C., 1934. The life-forms of plants and statistical plant geography. University Press, Oxford.
RUSIŃSKA H., RATYŃSKA H., Galera H. 1996. Tropical mosses in the greenhouses of the Botanical Garden of the Polish Academy of Sciences in Warszawa-Powsin. Fragm. Flor. Geobot. 4.2: 1030-1033.

SŁOWNIK BOTANICZNY. 1993. A.J. Szweykowscy (eds.). Wiedza Powszechna, Warszawa.

SUDNIK-WÓJCIKOWSKA B. 1987. Flora miasta Warszawy i jej przemiany w ciągu XIX i XX wieku. Część 1., Część 2. Dokumentacja. Wyd. Uniw. Warsz., Warszawa.

TRZCIŃSKA-TACIK H. 1971. Interesujące gatunki we florze ruderalnej miasta Krakowa. Materiały Zakładu Fitosocjologii Stosowanej Uniwersytetu Warszawskiego 27: 245-250.

TRZCIŃSKA-TACIK H. 1979. Flora synantropijna Krakowa. Rozprawy habilitacyjne Uniwersytetu Jagiellońskiego 32.

TUTIN T.G., HEYWOOD V.H., BURGES N.A., VALENTINE D.H., WALTERS S.M., WEBB D.A. 1964. Flora Europaea, v 1. University Press, Cambridge.

TUTIN T.G., HEYWOOD V.H., BURGES N.A., MOORE D.M., VALENTINE D.H., WALTERS S.M., WEBB D.A. 1968. Flora Europaea, v. 2. Cambridge University Press, Cambridge.

TUTIN T.G., HEYWOOD V.H., BURGES N.A., MOORE D.M., VALENTINE D.H., WALTERS S.M., WEBB D.A. 1972. Flora Europaea, v. 3. Cambridge University Press, Cambridge.

TUTIN T.G., HEYWOOD V.H., BURGES N.A., MOORE D.M., VALENTINE D.H., WALTERS S.M., WEBB D.A. 1976. Flora Europaea, v. 4. Cambridge University Press, Cambridge, London, New York, Melbourne.

TUTIN T.G., HEYWOOD V.H., BURGES N.A., MOORE D.M., VALENTINE D.H., WALTERS S.M., WEBB D.A. 1980. Flora Europaea, v. 5. Cambridge University Press, Cambridge, London, New York, New Rochelle, Melbourne, Sydney.

WIŚNIEWSKI J., ASPROU V. 1974. Chwasty w uprawach szklarniowych m. Łodzi. Zesz. Nauk. U Ł, Nauki Mat.-Przyr., Ser. 2, 54: 47-57.

ZARZYCKI K. 1984. Ekologiczne liczby wskaźnikowe roślin naczyniowych Polski. Inst. Botaniki PAN, Kraków.

\section{FLORA CHWASTÓW \\ W SZKLARNIACH OGRODU BOTANICZNEGO PAN W POWSINIE}

\section{STRESZCZENIE}

Na podstawie systematycznie prowadzonych badań w szklarniach Ogrodu Botanicznego - Centrum Zachowania Różnorodności Botanicznej Polskiej Akademii Nauk stwierdzono, że flora chwastów jest bardzo bogata: zanotowano łącznie 243 taksony roślin naczyniowych oraz 17 gatunków mszaków. Uwzględniając podział flory roślin naczyniowych pod względem pochodzenia i stopnia zadomowienia, stwierdzono współdominację elementu rodzimego (89 gatunków), grupy uciekinierów z upraw szklarniowych (87 taksonów) oraz trwale zadomowionych roślin obcego pochodzenia (48 gatunków). Uciekinierzy z upraw prowadzonych w sąsiedztwie szklarni to element flory liczący 10 taksonów. Na uwagę zasługują dwa gatunki przypadkowo zawleczone do szklarni wraz z materiałem roślinnym - jest to kategoria najmniej liczna, lecz jej przedstawicieli notowano bardzo często. Obserwacje prowadzone w otoczeniu powsińskich cieplarni wskazują, że oddziaływania pomiędzy badaną florą a roślinami występującymi w sąsiedztwie mają charakter jednostronny. W Powsinie nie zanotowano udowodnionego przypadku ekspansji roślin poza cieplarnie, natomiast przepływ diaspor na badany teren jest częstym zjawiskiem - wśród chwastów szklarniowych przeważają rośliny występujące również w bezpośrednim sąsiedztwie (147 gatunków). Do najistotniejszych czynników mających wpływ na zestaw chwastów występujących w cieplarniach należą: flora terenów sąsiadujących oraz sposób użytkowania szklarni (w tym rodzaj upraw).

SŁOWA KLUCZOWE: flora synantropijna, chwasty, analizy florystyczne, Ogród Botaniczny Polskiej Akademii Nauk. 